\title{
Search for the Invisible Decay of Neutrons with KamLAND
}

T. Araki, ${ }^{1}$ S. Enomoto, ${ }^{1}$ K. Furuno, ${ }^{1}$ Y. Gando, ${ }^{1}$ K. Ichimura, ${ }^{1}$ H. Ikeda, ${ }^{1}$ K. Inoue,${ }^{1}$ Y. Kishimoto, ${ }^{1}$ M. Koga, ${ }^{1}$ Y. Koseki, ${ }^{1}$ T. Maeda, ${ }^{1}$ T. Mitsui, ${ }^{1}$ M. Motoki, ${ }^{1}$ K. Nakajima, ${ }^{1}$ K. Nakamura, ${ }^{1}$ H. Ogawa, ${ }^{1}$ M. Ogawa, ${ }_{1}$ K. Owada, ${ }^{1}$ J.-S. Ricol, ${ }^{1}$ I. Shimizu, ${ }^{1}$ J. Shirai, ${ }^{1}$ F. Suekane, ${ }^{1}$ A. Suzuki, ${ }^{1}$ K. Tada, ${ }^{1}$ S. Takeuchi,${ }^{1}$ K. Tamae, ${ }^{1}$ Y. Tsuda, ${ }^{1}$ H. Watanabe,${ }^{1}$ J. Busenitz, ${ }^{2}$ T. Classen, ${ }^{2}$ Z. Djurcic, ${ }^{2}$ G. Keefer, ${ }^{2}$ D. S. Leonard, ${ }^{2}$ A. Piepke, ${ }^{2}$ E. Yakushev, ${ }^{2,}{ }^{*}$ B. E. Berger, ${ }^{3}$ Y. D. Chan, ${ }^{3}$ M. P. Decowski, ${ }^{3}$ D. A. Dwyer, ${ }^{3}$ S. J. Freedman, ${ }^{3}$ B. K. Fujikawa, ${ }^{3}$ J. Goldman, ${ }^{3}$ F. Gray, ${ }^{3}$ K. M. Heeger, ${ }^{3}$ L. Hsu, ${ }^{3}$ K. T. Lesko, ${ }^{3}$ K.-B. Luk, ${ }^{3}$ H. Murayama, ${ }^{3}$ T. O'Donnell, ${ }^{3}$ A. W. P. Poon, ${ }^{3}$ H. M. Steiner, ${ }^{3}$ L. A. Winslow, ${ }^{3}$ C. Jillings, ${ }^{4}$ C. Mauger, ${ }^{4}$ R. D. McKeown, ${ }^{4}$ P. Vogel, ${ }^{4}$ C. Zhang, ${ }^{4}$ C. E. Lane, ${ }^{5}$ T. Miletic,${ }^{5}$ G. Guillian, ${ }^{6}$ J. G. Learned, ${ }^{6}$ J. Maricic, ${ }^{6}$ S. Matsuno, ${ }^{6}$ S. Pakvasa, ${ }^{6}$ G. A. Horton-Smith ${ }^{7}$ S. Dazeley ${ }^{8}$ S. Hatakeyama, ${ }^{8}$ A. Rojas,${ }^{8}$ R. Svoboda ${ }^{8}$ B. D. Dieterle, ${ }^{9}$ J. Detwiler, ${ }^{10}$ G. Gratta, ${ }^{10}$ K. Ishii, ${ }^{10}$ N. Tolich,,${ }^{10}$ Y. Uchida,${ }^{10}$ M. Batygov, ${ }^{11}$ W. Bugg,,${ }^{11}$ Y. Efremenko, ${ }^{11}$ Y. Kamyshkov, ${ }^{11}$ A. Kozlov, ${ }^{11}$ Y. Nakamura, ${ }^{11}$ H. J. Karwowski, ${ }^{12}$ D. M. Markoff, ${ }^{12}$ R. M. Rohm, ${ }^{12}$ W. Tornow, ${ }^{12}$ R. Wendell, ${ }^{12}$ M.-J. Chen, ${ }^{13}$ Y.-F. Wang, ${ }^{13}$ and F. Piquemal ${ }^{14}$

\section{(KamLAND Collaboration)}

\author{
${ }^{1}$ Research Center for Neutrino Science, Tohoku University, Sendai 980-8578, Japan \\ ${ }^{2}$ Department of Physics and Astronomy, University of Alabama, Tuscaloosa, Alabama 35487, USA \\ ${ }^{3}$ Physics Department, University of California at Berkeley and Lawrence Berkeley National Laboratory, \\ Berkeley, California 94720, USA \\ ${ }^{4}$ W. K. Kellogg Radiation Laboratory, California Institute of Technology, Pasadena, California 91125, USA \\ ${ }^{5}$ Physics Department, Drexel University, Philadelphia, Pennsylvania 19104, USA \\ ${ }^{6}$ Department of Physics and Astronomy, University of Hawaii at Manoa, Honolulu, Hawaii 96822, USA \\ ${ }^{7}$ Department of Physics, Kansas State University, Manhattan, Kansas 66506, USA \\ ${ }^{8}$ Department of Physics and Astronomy, Louisiana State University, Baton Rouge, Louisiana 70803, USA \\ ${ }^{9}$ Physics Department, University of New Mexico, Albuquerque, New Mexico 87131, USA \\ ${ }^{10}$ Physics Department, Stanford University, Stanford, California 94305, USA \\ ${ }^{11}$ Department of Physics and Astronomy, University of Tennessee, Knoxville, Tennessee 37996, USA \\ ${ }^{12}$ Triangle Universities Nuclear Laboratory, Durham, North Carolina 27708, USA
}

and Physics Departments at Duke University, North Carolina State University, and the University of North Carolina at Chapel Hill, North Carolina, USA

${ }^{13}$ Institute of High Energy Physics, Beijing 100039, People's Republic of China

${ }^{14}$ CEN Bordeaux-Gradignan, IN2P3-CNRS and University Bordeaux I, F-33175 Gradignan Cedex, France (Received 23 December 2005; published 17 March 2006)

\begin{abstract}
The Kamioka Liquid scintillator Anti-Neutrino Detector is used in a search for single neutron or twoneutron intranuclear disappearance that would produce holes in the $s$-shell energy level of ${ }^{12} \mathrm{C}$ nuclei. Such holes could be created as a result of nucleon decay into invisible modes (inv), e.g., $n \rightarrow 3 \nu$ or $n n \rightarrow 2 \nu$. The deexcitation of the corresponding daughter nucleus results in a sequence of space and timecorrelated events observable in the liquid scintillator detector. We report on new limits for one- and twoneutron disappearance: $\tau(n \rightarrow$ inv $)>5.8 \times 10^{29}$ years and $\tau(n n \rightarrow$ inv $)>1.4 \times 10^{30}$ years at $90 \%$ C.L. These results represent an improvement of factors of $\sim 3$ and $>10^{4}$ over previous experiments.
\end{abstract}

Baryon number violation is an important signal of physics beyond the standard model. Grand-unified theories suggest that processes such as $p \rightarrow e^{+} \pi^{0}$ and $n \rightarrow K^{0} \bar{\nu}$ are important but suppressed by powers of the grand unification scale. However, baryon number violation is not limited to grand-unified theories. In fact, baryon number is an "accidental" symmetry arising from the pattern of particles in, and renormalizability of, the standard model. New particles or new physics such as supersymmetry or extra dimensions might also be the cause of baryon number violation. Moreover, the suppression may only be at the $\mathrm{TeV}$ scale, instead of the much higher grand-unified scale.

The Particle Data Group [1] lists more than 70 possible modes of nucleon decay which conserve electric charge, energy momentum, and angular momentum. Experimental lifetime limits of $10^{30}$ years for all but a few of these modes have been obtained. The decay modes with the poorest limits are so-called "invisible" modes, such as $n \rightarrow \nu$ 's and $n n \rightarrow \nu$ 's. Invisible modes are dominant in some models [2]. 
Invisible decay modes involving bound neutrons are potentially detectable even though no energetic charged particles are produced directly in the decay. If we assume that the unobserved decay products carry away most of the neutron rest-mass energy, then the residual nucleus, ${ }_{\mathrm{Z}}^{\mathrm{A}-1} X$ or ${ }_{Z}^{A-2} X$, has a hole or holes in the previously occupied shell. Particles emitted in the nuclear deexcitation of the daughter are the experimental signature for the process. Detectability is independent of the specifics of the process as long as the rest-mass energy is carried away by the undetected particles.

The SNO Collaboration established the best single neutron disappearance limit of $\tau(n \rightarrow$ inv $)>1.9 \times 10^{29}$ years (90\% C.L.) by searching for the deexcitation $\gamma$ rays following neutron disappearance in ${ }^{16} \mathrm{O}$ [3]. The best limit for two-neutron disappearance, $\tau(n n \rightarrow$ inv $)>4.9 \times 10^{25} \mathrm{y}$ (90\% C.L.), was set by the Borexino Collaboration [4] by searching for possible decays of unstable nuclides resulting from $n n$ disappearance in ${ }^{12} \mathrm{C},{ }^{13} \mathrm{C}$, and ${ }^{16} \mathrm{O}$.

KamLAND, currently the world's largest lowbackground liquid scintillator (LS) detector (the LS composition is $\left.\mathrm{CH}_{1.97}\right)$, has a low energy threshold $(<1 \mathrm{MeV})$ and good energy and spatial resolution, making it well suited for neutron disappearance searches. The LS is contained in a 13-m-diameter transparent nylon-based balloon suspended in nonscintillating purified mineral oil. The scintillator is viewed by 132517 -inch Hamamatsu and 554 slower 20-inch Hamamatsu photomultiplier tubes (PMTs) mounted on the inner surface of an 18-m-diameter spherical stainless-steel tank. The inactive mineral oil buffer serves as passive shielding against external backgrounds such as ${ }^{208} \mathrm{Tl} \gamma$ rays coming from the PMT's glass and nearby rocks. A 3200 metric ton waterCherenkov detector surrounds the stainless-steel sphere, acting as a cosmic-ray muon veto. The muon rate in the central detector is $0.34 \mathrm{~Hz}$. Details of KamLAND can be found elsewhere [5].

In this study, we consider the disappearance of neutrons only from the fully occupied $s$ shell in ${ }^{12} \mathrm{C}$ leaving the daughter nucleus in a highly excited state. This results in subsequent deexcitation with the emission of secondary particles ( $p, n, d, \alpha, \gamma)$ and the possible further decay of the residual radioactive nuclei. The probabilities for the various deexcitation modes following one- and two-neutron disappearance in ${ }^{12} \mathrm{C}$ were estimated with a statistical nuclear model by Kamyshkov and Kolbe [6]. Some of these modes, producing a sequence of time-and-spacecorrelated events, are detectable in KamLAND. Here we report on a search for the four decay modes listed in Table I, each producing a correlated triple signal in the detector.

According to Ref. [6], neutrons produced in these modes have energies up to $45 \mathrm{MeV}$. In the LS, the first scintillation signal arises from neutron-proton elastic scattering, $4.4 \mathrm{MeV} \gamma$ rays from the inelastic neutron scattering from ${ }^{12} \mathrm{C}$, and detectable secondaries of other neutron interactions in the scintillator. Neutrons thermalize and capture, mostly on protons $\left(\tau_{\text {capture }} \sim 210 \mu \mathrm{s}\right)$, producing a second $2.2 \mathrm{MeV} \gamma$ ray signal. A third correlated signal comes from the $\beta^{+}$decay of the residual nuclei $\left({ }^{10} \mathrm{C},{ }^{9} \mathrm{C}\right.$, or $\left.{ }^{8} \mathrm{~B}\right)$. The correlated triple signal is a powerful tool for identifying these decays in the presence of backgrounds.

The limits presented here are based on data collected between March 5, 2002 and October 31, 2004. Initially, KamLAND had 17-inch PMTs only operational with 22\% photocathode coverage and $7.3 \% / \sqrt{E(\mathrm{MeV})}$ energy resolution. After February 27, 2003 the photocathode coverage increased to $34 \%$ by including 20 -inch PMTs, which improved the energy resolution to $6.2 \% / \sqrt{E(\mathrm{MeV})}$. The location of the event in the detector is reconstructed from the arrival times measured with the PMTs. Typical spatial resolution for the reconstruction procedure at the detector center, for a pointlike energy deposition, is $\sim 20.6 \mathrm{~cm}$ $(\sim 24.0 \mathrm{~cm}) / \sqrt{E(\overline{M e V})}$ with 20 -inch (without 20-inch) PMT data. The energy of the event is reconstructed using the number of detected photoelectrons after correcting for position and PMT gain variations. The response of the detector is studied and monitored with periodic deployments of $\gamma$ ray $\left({ }^{203} \mathrm{Hg},{ }^{68} \mathrm{Ge},{ }^{65} \mathrm{Zn}\right.$, and $\left.{ }^{60} \mathrm{Co}\right)$ and neutron sources $(\mathrm{Am}-\mathrm{Be})$ along the central vertical axis of the detector. Natural radio isotopes remaining in the detector materials such as ${ }^{40} \mathrm{~K}$ and bismuth-polonium from $\mathrm{U} / \mathrm{Th}$ chains are also used for calibrations. Because of the external $\gamma$ ray background, a radial fiducial volume cut of $5 \mathrm{~m}$ $(5.5 \mathrm{~m})$ for the $n(n n)$ disappearance search is applied. This corresponds to a fiducial mass of 408.5 (543.7) metric tons, corresponding to $3.48 \times 10^{31}\left(4.63 \times 10^{31}\right) s$-shell neutrons in ${ }^{12} \mathrm{C}$ nuclei. The total livetime, after correction for muon-associated cuts to avoid backgrounds due to

TABLE I. Branching ratios for the ${ }^{11} \mathrm{C}^{*}\left({ }^{10} \mathrm{C}^{*}\right)$ [6] deexcitation modes after neutron (two-neutron) disappearance from the $s_{1 / 2}$ state in ${ }^{12} \mathrm{C}$ and the experimental signature (number of time-correlated hits) for observation of these modes in KamLAND.

\begin{tabular}{lccc}
\hline \hline Decay mode & Daughter $\left(\right.$ decay, $T_{1 / 2}$, and $\left.Q_{E C}\right)$ & Branching ratio \% & Exp. sign. (hits) \\
\hline$(n 1){ }^{11} \mathrm{C}(n)$ & ${ }^{10} \mathrm{C}_{\mathrm{gs}}\left(\beta^{+} ; 19.3 \mathrm{~s}, 3.65 \mathrm{MeV}\right)$ & 3.0 & 3 \\
$(n 2){ }^{11} \mathrm{C}(n, \gamma)$ & ${ }^{10} \mathrm{C}_{\mathrm{gs}}\left(\beta^{+} ; 19.3 \mathrm{~s}, 3.65 \mathrm{MeV}\right)$ & 2.8 & 3 \\
$(n n 1){ }^{10} \mathrm{C}(n)$ & ${ }^{9} \mathrm{C}\left(\beta^{+}, 0.127 \mathrm{~s}, 16.5 \mathrm{MeV}\right)$ & 6.2 & 3 \\
$(n n 2){ }^{10} \mathrm{C}(n, p)$ & ${ }^{8} \mathrm{~B}\left(\beta^{+} \alpha, 0.77 \mathrm{~s}, 18 \mathrm{MeV}\right)$ & 6.0 & 3 \\
\hline \hline
\end{tabular}


TABLE II. Top section: selection criteria used in the neutron (two-neutron) disappearance search. Bottom section: detection efficiency $\epsilon$ for the decay modes $n 1, n 2, n n 1$, and $n n 2$ given in Table I.

\begin{tabular}{lcc}
\hline \hline Quantity & $n$ disappearance & $n n$ disappearance \\
\hline$R_{1,2,3}[\mathrm{~m}]$ & 5.0 & 5.5 \\
$R_{X Y 3}[\mathrm{~m}]$ & $>1.0$ & $>1.0$ \\
$\Delta R_{12}[\mathrm{~m}]$ & 2.0 & 2.0 \\
$\Delta R_{13}[\mathrm{~m}]$ & 0.8 & 1.0 \\
$\Delta T_{12}[\mu \mathrm{s}]$ & $0.5-1000$ & $0.5-1000$ \\
$\Delta T_{13}[\mathrm{~s}]$ & $0.003-70$ & $0.003-6$ \\
$E_{1}[\mathrm{MeV}]$ & $0.9-25$ & $0.9-40$ \\
$E_{2}[\mathrm{MeV}]$ & $1.8-2.6$ & $1.8-2.6$ \\
$E_{3}[\mathrm{MeV}]$ & $1.5-3.8$ & $3.1-18.0$ \\
$\epsilon_{n 1(n n 1)}$ & $0.430 \pm 0.027$ & $0.680 \pm 0.032$ \\
$\epsilon_{n 2(n n 2)}$ & $0.651 \pm 0.033$ & $0.678 \pm 0.032$ \\
\hline \hline
\end{tabular}

muon-spallation events, is 749.8 days (751.4 days) for the $5.0 \mathrm{~m}(5.5 \mathrm{~m})$ fiducial volume.

The correlated triple event-selection cuts are summarized in Table II. The indices refer to the first, second, and third energy deposits (hits) in each candidate event. $R$ is the radial position of the hit, $R_{X Y}$ is the distance to the vertical central axis, $E$ is the reconstructed energy of the hit, $\Delta R$ and $\Delta T$ are the position and time differences between the hits. After applying all cuts to the data set, the number of observed $n(n n)$ disappearance candidate events is $1(0)$, compatible with the expected background, as discussed next.

No other process is expected to produce triple events with sufficient rates to result in backgrounds for the present search. Backgrounds to the $n$ and $n n$ disappearance search originate from doubly correlated signatures, such as those derived from reactor $\bar{\nu}_{e}$ events or other processes such as neutrons produced in ${ }^{13} \mathrm{C}(\alpha, n){ }^{16} \mathrm{O}$ followed by an uncorrelated coincidence within the selection cuts. The third uncorrelated hit is dominated by the tail of the ${ }^{210} \mathrm{Bi} \beta$ spectrum and ${ }^{208} \mathrm{Tl} \gamma$ rays [see Figs. 1(d) and 2(d)]. The data analysis cuts were optimized to suppress the accidental coincidence background and to improve the signal/ background ratio. For the case of the $n$ disappearance search, Fig. 1(a) shows the location of the accidental background events within the $5.5 \mathrm{~m}$ fiducial volume using a relaxed $\Delta R_{13}<2 \mathrm{~m}$ cut. Events inside the $5 \mathrm{~m}$ fiducial volume and outside of the $1 \mathrm{~m}$ cylinder cut around the central axis (to remove background from thermometers positioned at $0 \mathrm{~m}$ and $\pm 5.5 \mathrm{~m}$ ) are plotted in Figs. 1(b)1(d)] to show the position of the selected cuts. Similarly, Fig. 2 illustrates the selection criteria for the $n n$ disappearance search. To remove the background from muon induced neutrons and after-muon detector activity, a $2 \mathrm{~ms}$ veto of the entire detector volume following a muon traversal is applied for the case of the 3 rd hit. The $\beta$-neutron emitter ${ }^{9} \mathrm{Li}$ is produced by muon spallation and can give
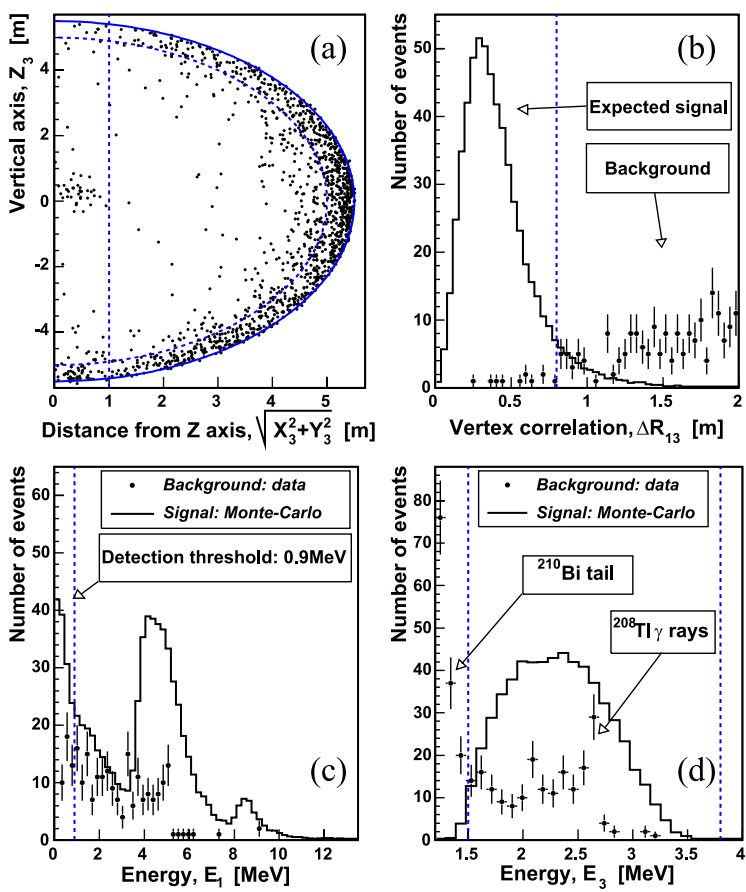

FIG. 1 (color online). $n$ disappearance search: (a) correlated triple background events in the 150-1000 s off-time window for the 3rd hit inside the $5.5 \mathrm{~m}$ detector radius; (b) -(d) events inside the $5 \mathrm{~m}$ fiducial volume and outside of the $1 \mathrm{~m}$ cylinder cut around the central axis are compared to the expected signal for $\tau=4 \times 10^{27} \mathrm{y}$, generated with the Monte Carlo simulation. Dashed lines indicate cuts used in the data analysis.

prompt and delayed signals. This background is suppressed by a $2 \mathrm{~s}$ veto within a $3 \mathrm{~m}$ radius cylinder around a wellreconstructed muon track. The entire volume is vetoed for $2 \mathrm{~s}$ following energy depositions with more than $10^{6}$ photoelectrons above what is expected from a minimum ionizing particle or if the muon is not well reconstructed. For $n$ disappearance the accidental background is estimated with a $150 \mathrm{~s}$ to $1000 \mathrm{~s}$ delayed-coincidence off-time window for the 3rd hit. In the $n n$ disappearance case a $10 \mathrm{~s}$ to $1000 \mathrm{~s}$ window is used. The number of expected accidental background events in the entire data set for the $n$ disappearance search is $0.82 \pm 0.26$. For the $n n$ disappearance search, the significantly greater energy of the 3rd hit [see Fig. 2(d)] allows selection of the energy threshold cut to be larger than the energy of the main source of background; the number of expected accidentals is $0.018 \pm 0.010$ events.

The efficiency of the position and energy cuts is calculated using a Monte Carlo procedure based on the GEANT code [7] set up to simulate the KamLAND detector response to the decay of the ${ }^{11} \mathrm{C}^{*}\left({ }^{10} \mathrm{C}^{*}\right)$ nuclear system. The code includes new libraries [8] which give a more accurate description (compared to standard GEANT) of the $(n, \gamma)$ and $\left(n, n^{\prime} \gamma\right)$ reactions. The decay schemes of ${ }^{10} \mathrm{C}$ and ${ }^{8} \mathrm{~B}$ are well known; the ${ }^{9} \mathrm{C}$ decay scheme is less well studied and the most recent data [9] are utilized for our simulations. The observed nonlinear dependence of the energy estima- 

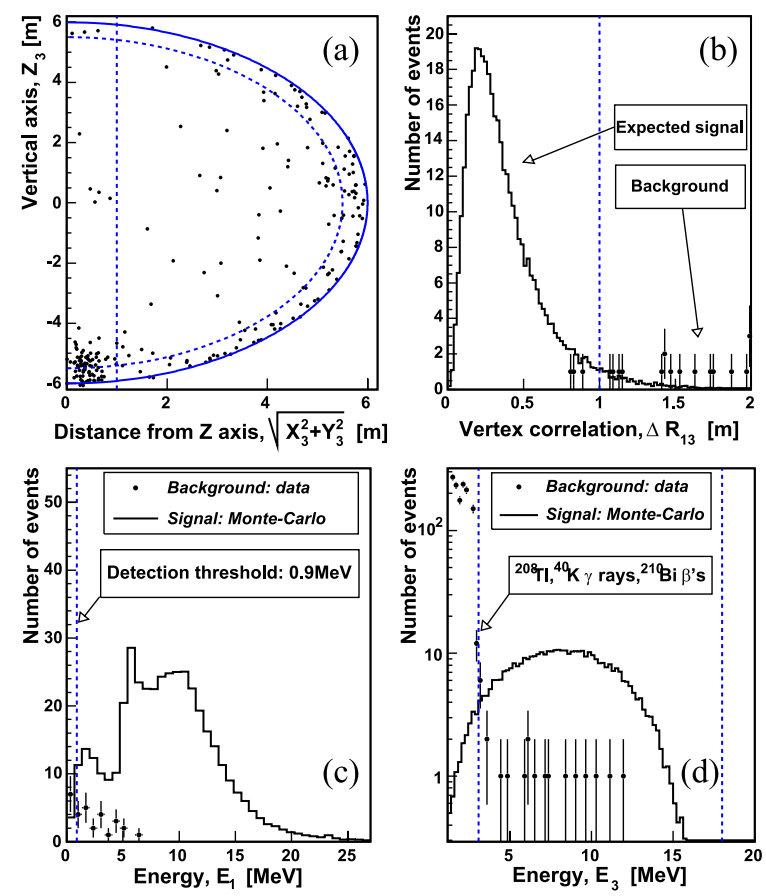

FIG. 2 (color online). $\quad n n$ disappearance search: (a) correlated triple background events in the 10-1000 s off-time window for the 3rd hit inside $6 \mathrm{~m}$ detector radius; (b)-(d) events inside the $5.5 \mathrm{~m}$ fiducial volume and outside of the $1 \mathrm{~m}$ cylinder cut around the central axis are compared to the expected signal for $\tau=$ $10^{28} \mathrm{y}$, generated with the Monte Carlo simulation. Dashed lines indicate cuts used in the data analysis.

tion upon particle energy and type (for $\gamma$ rays and the neutron Am-Be source) is reproduced with a combination of a correction for scintillator light output quenching [10] and a theoretical correction for Cherenkov light emission for electrons and positrons [Fig. 3(a)]. The energy and position are taken from the Monte Carlo simulation and then smeared according to the resolution observed in calibration $\gamma$ ray and other data [Fig. 3(b)]. The reliability of neutron simulations in the LS was tested by comparison to
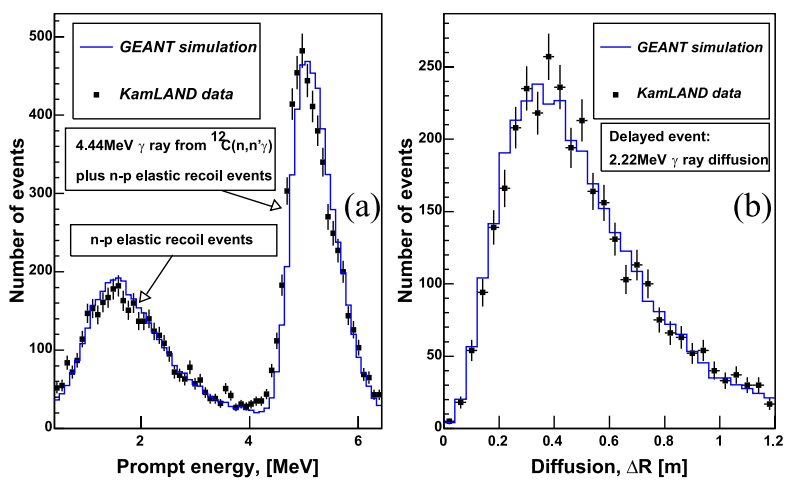

FIG. 3 (color online). KamLAND Am-Be data compared to the GEANT detector simulation: (a) prompt energy signal; (b) diffusion of the $2.22 \mathrm{MeV}$ delayed $\gamma$ ray from neutron capture on proton. simulations using the SCINFUL code [11] and a good agreement was found. The calculated efficiencies for the deexcitation modes in Table I are given in Table II.

The systematic uncertainty of the predicted theoretical nuclear deexcitation branching ratios as estimated in [6] is rather large, $\pm 30 \%$, while the experimental systematic uncertainties, such as the fiducial volume uncertainty and the uncertainty in neutron energy scale estimated by comparison of the Am-Be data and GEANT simulations [see also Table II and Ref. [5] ], are significantly smaller. The total systematic uncertainty is $\sim 30 \%$. The lifetime limits $\tau[n(n n) \rightarrow$ inv $]$ for $n$ and $n n$ disappearance are calculated using

$$
\tau[n(n n) \rightarrow \operatorname{inv}]>\frac{N_{0} T}{x_{\lim }} \sum_{i=1,2} \epsilon_{n(n n) i} B_{n(n n) i},
$$

where $N_{0}$ is the number of $s$-shell neutrons (or $n$ pairs), $T$ is the detector livetime and $\epsilon_{n(n n) i}$ is the detection efficiency (given in Table II), $B_{n(n n) i}$ is the branching ratio from Table I, and $x_{\lim }$ is the largest number of events compatible with observation at a given confidence level. If classical confidence intervals for Poisson processes with background [as described in Sec. II-III of Ref. [12]] are used, $x_{\lim }=3.07\left(x_{\lim }=2.28\right)$ events at $90 \%$ C.L., for neutron (two-neutron) disappearance, respectively. The corresponding lifetime limits at $90 \%$ C.L. are $\tau(n \rightarrow$ inv) $>7.2 \times 10^{29} \mathrm{y}$ and $\tau(n n \rightarrow$ inv $)>1.7 \times 10^{30} \mathrm{y}$. If the Feldman-Cousins procedure [12] is used instead of the simple Poisson limit, $x_{\text {lim }}=3.53$ events at $90 \%$ C.L. $\left(x_{\lim }=2.41\right.$ events at $90 \%$ C.L. $)$ and the corresponding lifetime limits are slightly reduced: $\tau(n \rightarrow$ inv $)>6.3 \times$ $10^{29} \mathrm{y}$ and $\tau(n n \rightarrow$ inv $)>1.6 \times 10^{30} \mathrm{y}$.

The effect of the uncertainty in the theoretical branching ratios and uncertainty for the number of expected background events is estimated using the program POLE [13]. Calculations using POLE are consistent with the results derived using the Feldman-Cousins procedure in the case of no systematic uncertainties and give $x_{\lim }=3.82$ events at $90 \%$ C.L. $\left(x_{\lim }=2.75\right.$ events at $90 \%$ C.L. $)$ when the systematic uncertainties are included; the lifetime limits are:

$$
\begin{gathered}
\tau(n \rightarrow \text { inv })>5.8 \times 10^{29} \mathrm{y} \text { at } 90 \% \text { C.L. } \\
\tau(n n \rightarrow \text { inv })>1.4 \times 10^{30} \mathrm{y} \text { at } 90 \% \text { C.L. }
\end{gathered}
$$

To summarize, about 838 metric ton years (1119 metric ton years) of KamLAND data have been analyzed in order to search for disappearance of a single neutron (a neutron pair) from the $s$ shell in ${ }^{12} \mathrm{C}$ which create subsequent nuclear deexcitations leading to three time and space correlated events. The observed number of events is consistent with that expected from the accidental coincidence background. This results in new improved limits on single neutron and two-neutron invisible decay lifetimes. This KamLAND result is a factor of 3 better than the pres- 
ent best limit from the SNO Collaboration [3] for $n$ disappearance, and more than 4 orders of magnitude better than the $n n$ disappearance limit set by the Borexino Collaboration [4].

The KamLAND experiment is supported by the COE program under Grant No. 09CE2003 of the Japanese Ministry of Education, Culture, Sports, Science, and Technology, and under the United States Department of Energy Grant No. DEFG03-00ER41138. We are grateful to the Kamioka Mining and Smelting Company that provided service for activities in the mine. We are also grateful to Scott Outten (University of Tennessee) for providing SCINFUL simulations.

*Present address: DLNP, JINR, Dubna, Russia.

[1] S. Eidelman et al. (Particle Data Group), Phys. Lett. 592, 1 (2004); http://pdg.lbl.gov.

[2] R. N. Mohapatra and A. Perez-Lorenzana, Phys. Rev. D 67, 075015 (2003).
[3] S. N. Ahmed et al. (SNO Collaboration), Phys. Rev. Lett. 92, 102004 (2004).

[4] H. O. Back et al. (Borexino Collaboration), Phys. Lett. B 563, 23 (2003).

[5] K. Eguchi et al. (KamLAND Collaboration), Phys. Rev. Lett. 90, 021802 (2003); T. Araki et al. (KamLAND Collaboration), Phys. Rev. Lett. 94, 081801 (2005).

[6] Y. Kamyshkov and E. Kolbe, Phys. Rev. D 67, 076007 (2003).

[7] J. Apostolakis, GEANT - Detector Description and Simulation Tool, http://wwwasd.web.cern.ch/wwwasd/geant/ index.html.

[8] C. Marquet et al., Nucl. Instrum. Methods Phys. Res., Sect. A 457, 487 (2001); I. Stekl et al., Nucl. Instrum. Methods Phys. Res., Sect. A 493, 199 (2002).

[9] L. Buchmann et al., Phys. Rev. C 63, 034303 (2001).

[10] J.B. Birks, Proc. Phys. Soc. London, Sect. A 64, 874 (1951).

[11] J. K. Dickens, SCINFUL code, ORNL-6462, 1988.

[12] G. J. Feldman and R. D. Cousins, Phys. Rev. D 57, 3873 (1998).

[13] J. Conrad et al., Phys. Rev. D 67, 012002 (2003). 